\title{
Enfermedad Diverticular. ¿Cuándo operar en forma electiva?
}

\author{
Alejandro Canelas \\ Hospital Alemán - Centro Privado de Cirugía y Coloproctología - HIGA Prof. Dr. Ramón Carrillo, CABA, Argentina.
}

Aproximadamente el 40\% de la población occidental presenta divertículos colónicos a los 50 años y estos exhiben un $4 \%$ de riesgo de desarrollar diverticulitis en un período de 10 años (15\% como complicados). ${ }^{1-3}$ Superados los cuadros inflamatorios la indicación de realizar sigmoidectomía es clara cuando los mismos han dejado como secuela estenosis, fístulas o tumoraciones donde no es posible descartar patología neoplásica. Sin embargo, cuando la inflamación se supera sin secuela la indicación quirúrgica electiva es motivo de controversia y fue cambiando con el correr de los años. Históricamente la idea de operar en forma electiva a estos pacientes se basó en preceptos como eliminar el riesgo elevado de nuevas diverticulitis graves con posibilidad de perforación, alta morbilidad y necesidad de cirugías con ostomías. Otros preceptos dominantes por muchos años fueron que los pacientes menores a 50 años, así como los que presentaban múltiples diverticulitis, tenían un riesgo significativamente mayor de recurrir en forma complicada.,

Al momento de indicar la cirugía en este escenario debemos analizar la evidencia científica de estos viejos preceptos y contraponer el riesgo real de recurrencia complicada con la efectividad y riesgos de la sigmoidectomía electiva.

Chapman y col. ${ }^{6}$ en su publicación de 2006 cuestionaron la indicación quirúrgica de ese entonces, operar tras 2 episodios de diverticulitis. En contra del precepto que tras reiterados episodios inflamatorios la posibilidad de respuesta a tratamiento médico era baja y la mortalidad alta, este grupo mostró que de 330 pacientes evaluados con diverticulitis complicadas, solo 150 tenían antecedentes de episodios inflamatorios previos. Al analizar este último subgrupo se observó que aquellos con 1 o 2 episodios previos presentaban mayor riesgo de perforación y necesidad de cirugía con ostomía que los que tenían antecedentes de 3 o más episodios previos. En base a esto, los autores concluyen que la recomendación de indicar cirugía electiva tras dos episodios inflamatorios era tardía para muchos casos e innecesaria para otros, debido a que la mayoría de las diverticulitis complicadas se dan en pacientes sin antecedentes.

\footnotetext{
El autor del trabajo no presenta conflicto de interés.

Alejandro Canelas

acanelas@hospitalaleman.com

Recibido: marzo de 2020. Aceptado: abril de 2020.
}

Analizando la evidencia sobre recurrencia de diverticulitis resultan interesantes los resultados de la revisión llevada a cabo por Strate y col. ${ }^{7}$ donde analizando 25388 pacientes de 10 estudios calcularon una recurrencia de 189/1000 pacientes (IC95\% 185-193) a 5 años, de los cuales un $30 \%$ presentarán múltiples episodios. Durante este mismo estudio se informa que la recurrencia en menores de 50 años es 234/1000 (IC95\% 217-256) y en mayores de 50 años 166/1000 (IC95\% 163-174). Pero el punto que más aporta a este análisis es que las recurrencias como diverticulitis complicada fueron 42/1000 (IC95\% 30-54), con un riesgo de cirugía de urgencia de 43/1000 (IC95\% 41-46) y de ostomía de 22/1000 (IC95\% 21-25).

Es decir, al indicar la cirugía electiva estamos buscando prevenir una recurrencia global de 19\%, una recurrencia complicada de 4,2\% y una posibilidad de ostomía de 2,2\% a 5 años. Estos resultados debemos contraponerlos con la efectividad y complicaciones de la cirugía electiva.

Al analizar la efectividad de la cirugía, la sigmoidectomía con sección distal a nivel de recto superior elimina la zona de alta presión a nivel de unión recto-sigmoidea disminuyendo la posibilidad de repetir diverticulitis a un $2,8 \%$ con seguimiento a 6,5 años. ${ }^{8}$ Sin embargo, cuando la resección deja sigma distal remanente las posibilidades de recidiva ascienden a 12,5\%. El trabajo de Thaler y col. ${ }^{8}$ no ha registrado casos de reoperación por recurrencia sobre 236 pacientes analizados. Por otro lado, la sección proximal colónica debe realizarse en un sitio sin inflamación ni hipertrofia de capa muscular para disminuir las tasas de dehiscencia anastomótica no dando importancia a la eventual persistencia de divertículos proximales. ${ }^{9}$ Esta información confirma que una sigmoidectomía técnicamente correcta es efectiva en la prevención de diverticulitis.

Respecto a las complicaciones mayores asociadas a la sigmoidectomía electiva por episodios previos de diverticulititis, Strate y col. ${ }^{7}$ en su revisión informan un riesgo de 111/1000 pacientes (IC95\% 20-273) en cirugía abierta y 87/1000 (IC95\% 49-130) en cirugía laparoscópica en las primeras 3 semanas postoperatorias. En nuestra publicación de Hospital Alemán en 2016 la tasa de complicaciones mayores fue $4,1 \%$ en sigmoidectomías laparoscópicas por antecedente de diverticulitis previas; con tasa de dehiscencia anastomótica de 2,1\%. ${ }^{10}$

Analizando estos resultados podemos concluir que con la cirugía electiva en estos pacientes no se logra reducir el riesgo 


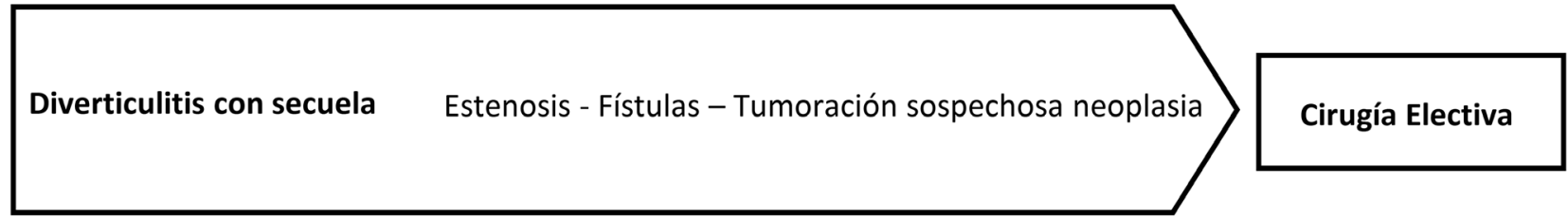

\begin{tabular}{|c|c|c|c|c|}
\hline $\begin{array}{l}\text { Diverticulitis no } \\
\text { complicada sin secuela }\end{array}$ & $\begin{array}{l}\text { Recurrencia } \\
\text { Recurrencia complicada } \\
\text { Riesgo de ostomía }\end{array}$ & $\begin{array}{l}\text { Evolución } \\
19 \% \\
4,2 \% \\
2,2 \%\end{array}$ & $\begin{array}{c}\text { Cx electiva } \\
2,8 \% \\
0 \% \\
2,1 \%\end{array}$ & $\begin{array}{l}\text { Analizar cada caso } \\
\text { Persistencia sintomática o } \\
>3 \text { episodios en } 2 \text { años }\end{array}$ \\
\hline $\begin{array}{l}\text { Diverticulitis complicada } \\
\text { sin secuela }\end{array}$ & $\begin{array}{l}\text { Recurrencia } \\
\text { Recurrencia complicada } \\
\text { Riesgo de ostomía }\end{array}$ & $\begin{array}{l}\text { Evolución } \\
28 \% \\
7 \% \\
1 \%\end{array}$ & $\begin{array}{c}\text { Cx electiva } \\
2,8 \% \\
0 \% \\
2,1 \%\end{array}$ & $\begin{array}{c}\text { Cirugía } \\
\text { Impacto + en calidad de } \\
\text { vida }\end{array}$ \\
\hline
\end{tabular}

Gráfico 1: Indicaciones de sigmoidectomía electiva por enfermedad diverticular.

de ostomía al paciente en forma significativa (graf. 1).

Ahora bien, es claro que aquellos pacientes con diverticulitis a repetición presentan un claro impacto en su calidad de vida. Este aspecto fue analizado en el trabajo DIRECT TRIAL, ${ }^{11}$ donde se incluyeron 109 pacientes con diverticulitis recurrente ( $\geq 3$ episodios en 2 años) o síntomas persistentes $\geq 3$ meses luego de un episodio inflamatorio y se los randomizó en: manejo conservador versus sigmoidectomía laparoscópica electiva. A los 6 meses de la randomización se evaluó el impacto en la calidad de vida (GIQLI) observando que los pacientes que se sometieron a cirugía tuvieron mayor score $(114,4+/-22,3$ vs. $100,4+/-22,7)$. De los pacientes de la rama tratamiento conservador, el $23 \%$ tuvieron que someterse a cirugía por aumento de síntomas abdominales. De la rama de pacientes operados, un 15\% presentó dehiscencia anastomótica. Considerando estos resultados los pacientes con persistencia sintomática y aquellos con más de 3 episodios de diverticulitis en 2 años mejorarían su calidad de vida con la cirugía.

Otro escenario a considerar es cómo manejar aquellos pacientes que han superado diverticulitis complicadas sin sigmoidectomía (abscesos manejados bajo tratamiento médico - drenaje percutáneo o peritonitis purulentas con lavado y drenaje). Si bien la mayoría de las guías de recomendación sugieren la sigmoidectomía electiva basado en la alta tasa de recurrencia y persistencia sintomática, muchos cuestionan esta indicación debido a que está basada en estudios retrospectivos, heterogéneos y con número limitado de pacientes. ${ }^{12,13}$ Resulta interesante la revisión sistemática de Lamb y Kaiser, ${ }^{14}$ donde analizando 1051 pacientes (22 estudios) se identificaron 739 que superaron diverticulitis complicada sin resección. Un 28\% de estos presentó recurrencia sintomática y $0,94 \%$ presentó perforación con peritonitis. Pero lo interesante es que se pudieron identificar 364 pacientes a los que nunca se les indicó cirugía electiva y estos presentaron un $18 \%$ de recurrencia sintomática con $0,5 \%$ de perforación libre. Lamentablemente incluye trabajos con número limitado de pacientes y seguimiento por tiempo acotado. Pero, a pesar de este sesgo, considerando estos resultados el mayor problema sería la recurrencia sintomática y no el desarrollo de complicaciones agudas, por lo cual es viable observar la evolución del paciente en el tiempo y considerar la indicación de cirugía electiva analizando cada caso.

Concluyendo, el gráfico 1 sintetiza las indicaciones de sigmoidectomía electiva por enfermedad diverticular colónica.

\section{BIBLIOGRAFÍA}

1. Peery A, Keku T, Martin C, Eluri S, Runge T, Galanko J, Sandler R. Distribution and characteristics of colonic diverticula in a United States screening population. Clin Gastroenterol Hepatol 2016; 14: 980-985.

2. Shahedi K, Fuller G, Bolus R, Cohen E, Vu M, Shah R, Agarwal N, Kaneshiro M, Atia M, Sheen V, Kurzbard N, van Oijen MG, Yen L, Hodgkins P, Erder MH, Spiegel B. Long-term risk of acute diverticulitis among patients with incidental diverticulosis found during colonoscopy. Clin Gastroenterol Hepatol 2013; 11: 1609-1613.

3. Ambrosetti P, Chautems R, Soravia C, Peiris-Waser N , Terrier F. Long-term outcome of mesocolic and pelvic diverticular abscesses of the left colon: a prospective study of 73 cases. Dis Colon Rectum 2005; 48: 787-791.

4. Chautems R, Ambrosetti P, Ludwig A, Mermillod B, Morel P, Soravia C. Long-term follow-up after first acute episode of sigmoid diverticulitis: is surgery mandatory?: a prospective study of 118 patients. Dis Colon Rectum 2002; 45: 962-966.

5. Roberts P, Abel M, Rosen L, Cirocco W, Fleshman J, Leff E, Levien 
D, Pritchard T, Wexner S, Hicks T, et al. Practice parameters for sigmoid diverticulitis. The Standards Task Force American Society of Colon and Rectal Surgeons. Dis Colon Rectum 1995; 38: 125132.

6. Chapman J, Dozois E, Wolff B, Gullerud R, Larson D. Diverticulitis: A Progressive Disease? Do Multiple Recurrences Predict Less Favorable Outcomes? Ann Surg 2006; 243: 876-883.

7. Strate L, Peery A, Neumann I. American Gastroenterological Association Institute Technical Review on the Management of Acute Diverticulitis. Gastroenterology 2015; 149: 1950-1976.

8. Thaler K, Baig M, Berho M, Weiss E, Nogueras J, Arnaud J, Wexner $\mathrm{S}$, Bergamaschi R. Determinants of recurrence after sigmoid resection for uncomplicated diverticulitis. Dis Colon Rectum 2003; 46: 385-388

9. Feingold D, Steele S, Lee S, Kaiser A, Boushey R, Buie D, Rafferty J. Practice Parameters for the Treatment of Sigmoid Diverticulitis. Dis Colon Rectum 2014; 57: 284-294.

10. Rotholtz N, Canelas A, Bun M, Laporte M, Sadava E, Ferrentino N, Guckenheimer S. Laparoscopic approach in complicated diverticular disease. World J Gastrointest Surg 2016; 8: 308-314.

11. van de Wall B, Stam M, Draaisma W, Stellato R, Bemelman W, Boermeester M, Broeders I, Belgers E, Toorenvliet B, Prins H, Consten E, on behalf of the DIRECT trial collaborators. Surgery versus conservative management for recurrent and ongoing leftsided diverticulitis (DIRECT trial): an open-label, multicentre, randomised controlled trial. Lancet Gastroenterol Hepatol 2017; 2: 13-22.

12. Galetin T, Galetin A, Vestweber K, Rink A. Systematic review and comparison of national and international guidelines on diverticular disease. Int J Colorectal Dis 2018; 33: 261-272.

13. Gaertner W, Willis D, Madoff R, Rothenberger D, Kwaan M, Belzer G, Melton G. Percutaneous drainage of colonic diverticular abscess: is colon resection necessary? Dis Colon Rectum 2013; 56: 622-626.

14. Lamb N, Kaiser K. Elective Resection Versus Observation After Nonoperative Management of Complicated Diverticulitis With Abscess: A Systematic Review and Meta-Analysis. Dis Colon Rectum 2014; 57: 1430-1440. 